\title{
Commentary: Coincidence or consequence, and the effect of sex on either
}

\author{
Berhane Worku, MD, and Mario Gaudino, MD
}

\author{
From the Department of Cardiothoracic Surgery, Weill Cornell Medical Center, New York, NY. \\ Disclosures: Authors have nothing to disclose with regard to commercial support. \\ Received for publication March 20, 2019; accepted for publication March 21, 2019; available ahead of print April \\ $20,2019$. \\ Address for reprints: Mario Gaudino, MD, Department of Cardiothoracic Surgery, Weill Cornell Medical Center, \\ 525 E 68th St, M-404, New York, NY 10065 (E-mail: mfg9004@med.cornell.edu). \\ J Thorac Cardiovasc Surg 2019;158:1081-2 \\ $0022-5223 / \$ 36.00$ \\ Copyright (C) 2019 by The American Association for Thoracic Surgery \\ https://doi.org/10.1016/j.jtcvs.2019.03.072
}

Acute kidney injury (AKI) is a well described complication after cardiac surgery with cardiopulmonary bypass (CPB), and it is associated with increased morbidity and mortality. A number of studies have described an association between the nadir hematocrit (nHct) during CPB and the risk of postoperative AKI. $^{1-4}$

In their study in this issue of the Journal, Brescia and colleagues $^{5}$ used a large multi-institutional database to analyze the effect of sex on the relationship between nHct during CPB and AKI in 17,873 patients undergoing isolated coronary artery bypass grafting. Women demonstrated a greater burden of preoperative comorbidities, including worse renal function. Women underwent blood conservation strategies less often and received more fluid administration (including prime volume and total fluid administered) and blood transfusions. Women had a lower preoperative hematocrit, a lower nHct during $\mathrm{CPB}$, and a lower postoperative hematocrit, and they also had more postoperative complications, including AKI and mortality. After adjustment for relevant variables, lower nHct was associated with AKI in the overall population (odds ratio, 0.96), and sex did not alter this association (lower nHct was associated with AKI in men and women [odds ratio, 0.96 for both] when analyzed separately ${ }^{5}$ ).

Brescia and colleagues ${ }^{5}$ are to be commended on the extensive statistical analysis that they performed on a large, high-quality data set, which is what makes such a study feasible. Only 2 previous studies had focused on answering this question. One study used the same data set and found that nHct was associated with AKI in men but not women undergoing all types of cardiac surgery. ${ }^{1}$ Men more frequently underwent coronary artery bypass grafting, however, potentially confounding the findings. In another study, nHct was associated with AKI in men and women, but women demonstrated a nonsignificantly lower, albeit

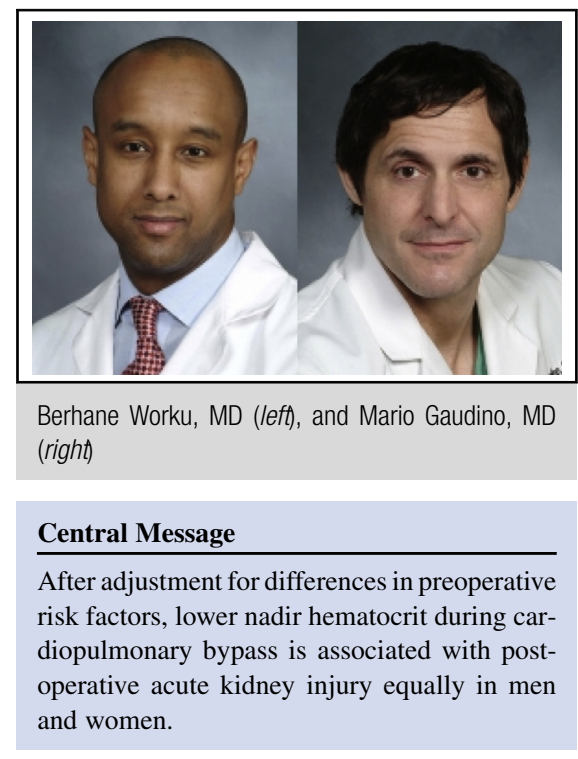

See Article page 1073.

numerically lower, rate of AKI in the subgroup of patients with severe hemodilution. ${ }^{2}$ It is suggested that menstruation may result in adaptive mechanisms in women that allow them to tolerate anemia better.

The etiology of AKI after CPB is multifactorial, with preoperative renal function, exposure to nephrotoxins, intraoperative hemodynamic fluctuations, the systemic inflammatory response syndrome, and postoperative cardiac and other complications likely all contributing factors. ${ }^{4}$ Given the inherent variability in the use of blood conservation strategies and in the management of intraoperative hemodynamics, including fluid and blood product administration and pharmacologic manipulation, as seen in the study of Brescia and colleagues, ${ }^{5}$ and given the imprecision of any statistical method to control for the effect of these factors on hematocrit and AKI, any retrospective study attempting to demonstrate an association between nHct and AKI is prone to confounding.

Until we prove that low nHct causes AKI, which would require a randomized trial, it is difficult to speculate on the second question, whether sex alters this interaction. Are we demonstrating the effect of sex on 2 coincidentally associated findings, in which case one would expect no effect, or on the consequence of one finding on the other? 
Further compounding the analysis of an interaction with sex is that women will almost certainly demonstrate lower $\mathrm{nHct}$ on CPB because women have lower preoperative baseline hematocrits, ${ }^{1,2}$ as seen in the study of Brescia and colleagues. ${ }^{5}$

That being said, it is likely that a true association exists. Methods to avoid excessive intraoperative hemodilution are therefore warranted, including such standard blood conservation strategies as retrograde autologous priming, ultrafiltration, and cardiotomy suction, as well as such less-standard methods as the use of mini-CPB circuits. The question of whether such a strategy should be preferentially applied to one sex more than the other, however, remains to be answered.

\section{References}

1. Ellis MC, Paugh TA, Dickinson TA, Fuller J, Chores J, Paone G, et al. Nadir hematocrit on bypass and rates of acute kidney injury: does sex matter? Ann Thorac Surg. 2015;100:1549-54; discussion 1554-5.

2. Mehta RH, Castelvecchio S, Ballotta A, Frigiola A, Bossone E, Ranucci M. Association of gender and lowest hematocrit on cardiopulmonary bypass with acute kidney injury and operative mortality in patients undergoing cardiac surgery. Ann Thorac Surg. 2013;96:133-40.

3. Karkouti K, Beattie WS, Wijeysundera DN, Rao V, Chan C, Dattilo KM, et al. Hemodilution during cardiopulmonary bypass is an independent risk factor for acute renal failure in adult cardiac surgery. J Thorac Cardiovasc Surg. 2005;129: 391-400.

4. Ranucci M, Aloisio T, Carboni G, Ballotta A, Pistuddi V, Menicanti L, et al. Acute kidney injury and hemodilution during cardiopulmonary bypass: a changing scenario. Ann Thorac Surg. 2015;100:95-100.

5. Brescia AA, Wu X, Paone G, Heung M, Paugh TA, Shann K, et al. Effect of sex on nadir hematocrit and rates of acute kidney injury in coronary artery bypass. $J$ Thorac Cardiovasc Surg. 2019;158:1073-80.e4. 